\title{
Una revisión crítica en la enseñanza universitaria de la arquitectura. El caso de la UNAM, México y la UNCUYO, Argentina
}

A Critical Review in University Teaching of Architecture. The Case of UNAM, Mexico and UNCUYO, Argentina

Uma revisão crítica do ensino universitário em arquitetura. O caso da UNAM, no México e UNCUYO, na Argentina

Virginia Miranda Gassull ${ }^{*}$

Recibido: 30 de abril del 2020

Aprobado: 24 de noviembre del 2020

https://doi.org/10.12804/revistas.urosario.edu.co/territorios/a.9030

Para citar este artículo:

Miranda Gassull, V. (2021). Una revisión crítica en la enseñanza universitaria de la Arquitectura. El caso de la UnAm, México y la UnCUYO, Argentina. Territorios, (44-Especial), 233-259. https://doi.org/10.12804/

* UNCUYO-CONICET, Mendoza, Argentina. Correo electrónico: arq. vmiranda@gmail.com ORCID: https://orcid. org/0000-0001-9382-5906 revistas.urosario.edu.co/territorios/a.9030 
Palabras clave

Universidad; epistemología; crítica; arquitectura; UNCUYO;

UNAM.

Keywords

University; epistemology; criticism; architecture; UNCUYO; UNAM.

Palavras-chave

Universidade; epistemologia; critica; arquitetura; UNCUYO;

UNAM.

\section{tersitarios 44 Especial}

RESUMEN

La universidad cumple un rol fundamental en la sociedad, específicamente, en la configuración del hábitat humano. Este artículo parte desde la Arquitectura como una disciplina que promueve prácticas, narrativas y espacialidades que modifican nuestra forma de habitar los territorios. Se problematiza la enseñanza de la arquitectura en la manera de ejercer la disciplina, a los habitantes que prioriza y la racionalización del espacio social. Se propone analizar la perspectiva socio-crítica en las escuelas de Arquitectura latinoamericana con el fin de reconocer el abordaje ontoepistémico y el diseño metodológico de las tres funciones de la universidad. Se toman como caso de estudio dos escuelas con antigüedades distintas y escalas urbanas diferentes (metrópolis y megalópolis). Como resultado, se exponen las propuestas pedagógicas de dos espacios académicos que abordan el tema del hábitat humano de manera integral desde la Arquitectura, como un proceso dialéctico entre las necesidades de la gente y la configuración de los territorios.

\section{ABSTRACT}

The university plays a fundamental role in society and specifically in the configuration of human habitat. It starts from Architecture as a discipline that promotes practices, narratives and spatialities that modify our way of inhabiting the territories. The teaching of architecture is problematized in the way of exercising discipline, the citizens it prioritizes and the rationalization of the social space. It is proposed to analyze the socio-critical perspective in Latin American Architecture schools in order to recognize the ontoepistemic approach and the methodological design of the three functions of the University. Two architecture schools with different antiquities and on different urban scales (metropolis and megalopolis) are taken as a case study. As a result, the pedagogical proposals of two academic spaces that address the subject of human habitat in an integral way from Architecture are exposed, as a dialectical process between the needs of people and the configuration of the territories.

\section{RESUMO}

A universidade desempenha um papel fundamental na sociedade e especificamente na configuração do habitat humano. Parte-se da arquitetura como uma disciplina que promove práticas, narrativas e espacialidades que modificam nossa forma de habitar os territórios. Se problematiza o ensino de arquitetura quanto à forma de exercer a disciplina, os habitantes que ela prioriza e a racionalização do espaço social. Propõe-se a analisar a perspectiva sociocrítica nas universidades de arquitetura latino-americanas a fim de reconhecer a abordagem ontoepistêmica e o desenho metodológico das três funções da universidade. São tomadas como um estudo de caso duas universidades de arquitetura de diferentes idades e em diferentes escalas urbanas (metrópole e megalópole). Como resultado, são expostas as propostas pedagógicas de dois espaços acadêmicos que abordam a questão do habitat humano de forma integral a partir da arquitetura, como um processo dialético entre as necessidades das pessoas e a configuração dos territórios. 


\section{Introducción}

En Latinoamérica, la educación superior o universitaria tiene como objetivo adecuarse a las profundas transformaciones sociales que se han producido en el siglo XXI, tales como las innovaciones tecnológicas, las crisis productivas, los avances científicos, las mutaciones en los patrones de asentamiento urbano-rurales, entre otras. Uno de los principales ejes del avance en educación superior apunta a promover un desarrollo sustentable y garantizar la justicia social e igualdad de oportunidades, que constituyen una dimensión fundamental que debe incidir en la definición de políticas educativas, científicas y tecnológicas para los próximos años (Tauber, 2010).

La universidad cumple un rol fundamental en la sociedad, específicamente, en la configuración del hábitat humano. Sin embargo, ha prevalecido una vinculación hacia sectores productivos, empresariales, y en el caso de la Arquitectura, se repite este patrón que favorece a los sectores de poder adquisitivo medio y alto. Esto se viene exponiendo desde los 70; como refiere la revista Los Libros en Córdoba, Argentina: "La institución universitaria tal como existe y desde sus orígenes responde a una concepción conservadora que la define como aparato legitimador, reproductor y consolidador del Estado en tanto expresión de la clase dominante" $(1971$, p. 7$)$. Existe entonces una dicotomía entre la producción del conocimiento universitario y la capacidad de responder a las realidades de los sectores populares. En esta línea, el investigador Barreto (2006) afirma:

La Arquitectura no termina de aceptar plenamente como propios los problemas del hábitat social y en particular los de la urbanización informal - que curiosamente son ampliamente reconocidos como competencia legal de los arquitectos- tratándolos en muchos casos de manera despectiva o como un tema ajeno a la profesión (p. 24).

El modelo universitario ha transitado diferentes patrones, según González y González (2003) algunos como el modelo tradicional (generador - y dueño- del conocimiento), el modelo economicista (facilitador y socio del campo productivo empresarial) y el modelo de desarrollo integral (función social y democratización del saber). En Latinoamérica, desde los 80 ha primado el modelo economicista que propone un vínculo difusionista-tranferencista (Tommassino \& Cano, 2016) basado en el circuito ciencia-innovaciónaplicación funcional al mercado, en el cual no importa la calidad del vínculo, sino difundir conocimiento y transferir tecnología como fin último. Como se viene exponiendo, en este modelo no se prioriza a los sectores con mayores problemáticas. En palabras de Maass y Sabulsky (2015): "Se trata de una lógica vinculacionista que alentó una dinámica unilateral, centrada territarios 44 Especial 235 


\section{territorias} 44 Especial en la transferencia de conocimientos de la universidad al medio" $(2015$, p. 86$)$.

La desarticulación entre sociedaduniversidad también ha tenido su impacto en la configuración de los territorios. En este sentido, se problematiza desde la enseñanza de la arquitectura y la compleja trama que ha dejado la modernidad en la forma de ejercer la disciplina, los habitantes que prioriza y la racionalización del espacio social. Este texto parte de la premisa que la Arquitectura tiene una estrecha relación en la configuración de los territorios, entendiéndola como una disciplina que promueve prácticas, narrativas y espacialidades que modifican nuestra forma de habitar el mundo.

En la formación de Arquitectura predomina un enfoque formalista y técnico para dar respuesta habitacional, esto se puede observar en la mirada "viviendista" y "esculturista" que predomina en la respuesta a este déficit (habitacional). Un enfoque que radica en la objetivación de la vivienda y de sus usuarios, es decir, los y las habitantes. El estudio del hábitat como un objeto divorciado de sus partes y del territorio, propone un campo de trabajo dividido en diferentes especialidades. A su vez, la arquitectura se ejerce desde la forma y la función como dimensiones centrales, en la que se descontextualiza el espacio-tiempo y, con ello, los procesos socioterritoriales. En esta línea los investigadores mexicanos Márquez López y Pradilla Cobos exponen:
Las grandes ciudades latinoamericanas aparecen hoy como totalidades fragmentadas y socialmente segregadas en las que se combinan, conflictivamente, de un lado, las más contemporáneas obras arquitectónico urbanas de los más famosos despachos de arquitectura del mundo, con alta tecnología, dotados de todos los servicios urbanos de calidad, para el funcionamiento del capital y la vivienda de los capitalistas y sus administradores públicos y privados; $\mathrm{y}$, de otro lado, los más sórdidos conventillos centrales o degradados tugurios periféricos carentes de todo, donde habitan los asalariados peor pagados, la sobrepoblación relativa, el lumpen proletariado; son las dos caras de la misma moneda producida por el capitalismo neoliberal salvaje en Latinoamérica (2016, p. 31).

La pobreza e informalidad es un hecho existente en toda Latinoamérica, y según Robinson (2006), "en todo el mundo" las ciudades globales más exitosas y económicamente productivas, generan informalidad y pobreza. Está claro que las formas de informalidad y pobreza urbana en el mundo son diferentes según los procesos sociourbanos que se han dado en la historia de las ciudades, pero la tesis que plantea Robinson despeja que ciertos "conceptos" sobre ciudades globales, ciudad-región, entre otros, no visibilizan la informalidad y pobreza del total de sus territorios. Así mismo, en este artículo se enfatiza sobre corrientes latinoamericanas que desenmascaran la informalidad desde 
posiciones críticas que proponen otras formas de saber-hacer y sitúan en primer plano a los sujetos de los territorios locales. La arquitectura no es ajena a este proceso y se esbozan aproximaciones de cómo se imparte la educación en escuelas de arquitectura desde una perspectiva crítica, latinoamericana y participativa.

En contraposición al modelo dominante en las escuelas de arquitectura, existe también dentro de la disciplina, la perspectiva epistemológica ligada al pensamiento crítico que se expande hacia el conocimiento co-construido socialmente que incorpora a otros sujetos $y$ diferentes formas de saber y hacer. A su vez, esta perspectiva supera la idea de "fábrica de profesionales" (Carlevaro, 2010) promoviendo procesos integrales del conocimiento desde las tres funciones sustantivas de la universidad (extensión, docencia e investigación).

En este sentido, se plantea la relación que tiene el conocimiento universitario como dispositivo esencial para garantizar el derecho a habitar dignamente la diversidad de la población. El pensamiento crítico de la arquitectura tiene como desafío la construcción de un cuerpo teórico que proponga (y habilite) nuevas formas de pensar el habitar en Latinoamérica. Cabe aclarar que, cuando se menciona la crítica en la arquitectura, no se refiere al método empleado comúnmente en la formación y práctica de la profesión de "criticar arquitectura" como juicio estético, medio de aprendizaje y creación - muy utilizado en los talleres de arquitectura-, sino que se refiere a la epistemología crítica que funda ideas sobre una arquitectura transformadora.

Desde esta perspectiva, se focalizó la atención en la formación de arquitectos insertos en la compleja trama del acceso a un hábitat digno, lo cual generó el interrogante principal: ¿los arquitectos y arquitectas están formados para abordar la realidad sociohabitacional?

Para responder a esta pregunta, se introducen los paradigmas dominantes en la formación en Arquitectura para luego analizar la perspectiva socio-crítica de las escuelas de arquitectura seleccionadas, en vista de reconocer el abordaje ontoepistémico y el diseño metodológico que integra las tres funciones de la universidad. Para ello, se toma como caso de estudio dos escuelas de arquitectura en el norte de Latinoamérica (UNAM) y el sur (UNCUYO), que tienen 100 años de diferencia respecto a la antigüedad de creación (1910 y el 2011, respectivamente) y en escalas urbanas diferentes (metrópolis y megalópolis). Dicha selección es resultado de un intercambio docente y posdoctoral entre dos espacios académicos ${ }^{1}$ de las universidades mencionadas.

Se analizan las diferentes corrientes epistemológicas de la arquitectura que han formado el pensamiento crítico en Latinoamérica a partir del estudio de fuentes secundarias, prevaleciendo la producción realizada desde Latinoamérica y por autores de la región.
1 Taller Max Cetto y la linea de investigación Arquitectura, Diseño, Complejidad y Participación (ADCP) y maestría en Arquitectura de la facultad de Arquitectura de la UNAM y la cátedra de Vivienda Social de la UNCUYO. 
${ }^{2}$ La carrera de arquitectura se fundó en la Academia de San Carlos en 1785 y la UNAM la beredó desde 1910.

\section{territorias 44 Especial}

Por otra parte, se realizaron cinco entrevistas en profundidad a investigadores del taller Max Cetto en la UNAM, como docentes de la línea de investigación en Arquitectura, Diseño, Complejidad y Participación (ADCP), doctorado y maestría en Arquitectura de la facultad de Arquitectura de la Universidad Nacional Autónoma de México. También se hicieron dos entrevistas en profundidad a docentes de la cátedra de Vivienda Social de la Universidad Nacional de Cuyo. En el caso de la UNCUYO (por su corta trayectoria) se complementó la información contextualizando a la carrera de Arquitectura (y su importancia) en el contexto nacional de universidades públicas. Se analizaron fuentes primarias y secundarias sobre las facultades de Arquitectura en Argentina y los espacios académicos seleccionados, combinando el análisis documental y comparativo en planes de estudio, programas de cátedra y perfil del egresado en instituciones públicas argentinas.

\section{El paradigma de la enseñanza en Arquitectura en Latinoamérica}

La enseñanza en Arquitectura comenzó en el siglo Xx. Las primeras escuelas de arquitectura en Latinoamérica datan desde 1901: la Escuela de Arquitectura UBA en Buenos Aires, convertida en facultad en 1947; la UNAM-México² (1910); la UNCCórdoba (1931); la FAUUSP (1948) en Sao Paulo; la UNLP (1952) en La Plata; entre otras. En ese marco, hay diversos autores que periodizan la enseñanza de la arquitectura en Latinoamérica y el pensamiento de la época, como Ramón Gutiérrez y Rodrigo Gutiérrez Viñuales (2012) y, Herna Lameda Luna (2014) en las siguientes etapas: de 1930 a 1945, inicio al modernismo; de 1945 a 1970, crisis del modernismo - y sus consecuencias-; y desde 1970 hasta la actualidad, es bastante discutido si denominarla posmodernidad, modernidad tardía, posestructuralismo o como la nombra Lameda Luna (2014, p. 654) y con quien se adhiere "autocrítica y revisión historicista”. En esta última época, se producen trasformaciones sobre la revisión de la arquitectura y sus procesos para la construcción del cuerpo teórico.

El modernismo se adopta en las escuelas de arquitectura en Latinoamérica en la primera etapa mencionada, a mitad del siglo XX, es decir, simultáneo a la creación de dichas escuelas. El planeamiento ahistoricista y descontextualizado que propone este modelo fue devastador para la construcción de las ideas de estas sociedades, en este caso, de la arquitectura de la región. De igual manera, se puede exponer que el modernismo no solo trajo las recetas racionalizadoras del espacio, de la vivienda con el hombre blanco heteronormado como estereotipo, sino que a su vez, promulgó respuestas seriadas al problema habitacional, pero negando el problema de la informalidad y la pobreza, sus orígenes, sus causas, y principalmente, la diversidad de sus habitantes. Como afirma Marina Waisman (1989, p. 10), "no puede existir 
una modernidad para pueblos desarrollados y otra para nosotros", la autora se pregunta si será una modernidad de segunda, y continúa esbozando: "La técnica en sí misma no puede erigirse en valores supremos, como tampoco puede aceptarse la exclusión como método" (p. 10). En esa perspectiva, el grupo de investigadores Castaño et al. expresan que "no ha existido una conciencia pedagógica en las escuelas de arquitectura. Por este motivo, las discusiones se han centrado en cuestiones de forma más que de fondo" (2005, p. 127).

El método empírico cartesiano de tradición racionalista es el nudo central del pensamiento moderno, sumado a la homogeneización y pulverización de los y las habitantes y la vida social colectiva. Como afirma García Ramírez (2012), "entre lo que los arquitectos pensamos y asumimos que es la vida en comunidad, y lo que ocurre en la realidad, existe una brecha que se ha venido ampliando con el paso del tiempo". En esta frase, se puede sintetizar lo que se viene mencionado: la arquitectura es un campo del conocimiento inminentemente social. Los enunciados arquitectura para la gente, arquitectura con la gente, arquitectura sin arquitectos $\mathrm{y}$, otros que existen en el ámbito de estudio, evidencian la objetivación existente entre la arquitectura y las necesidades de la sociedad. De ese modo, se resume cómo la arquitectura se ha convertido en una mediación del capital, en la cooptación de los arquitectos y las arquitectas en la construcción de un mundo ficticio útil a las corporaciones y no a la sociedad.

A partir de ello, resulta inminente repasar cuáles han sido las líneas que han discutido o, al menos, han aportado al pensamiento crítico de la región. Josep Montaner, en su libro Arquitectura y crítica (1999) hace un recorrido sobre los creadores del movimiento moderno y su impacto en las ciudades, en especial, en Latinoamérica. Según el autor, Walter Benjamín es uno de los autores clave para la crítica de la modernidad, en la que se desentraña el fundamento de autoría individual de la arquitectura. Al respecto, el autor expone que

se disuelve el mito clásico de la unicidad de la obra, que ahora pasa a ser depurada por las técnicas de reproducción, y se invalida para siempre el carácter de afirmación individual de un momento creativo que pasa a ser un trabajo colectivo [...] (Montaner, 1999, p. 69).

En Latinoamérica existen autores locales (o radicados) que han promovido el mundo de las ideas críticas a nivel regional como Roberto Segre, relator de la obra América Latina en su Arquitectura, la cual postula una mirada juiciosa sobre la dominación de la arquitectura racionalista en la que no existe lugar para lo singular, la participación y el valor de lo artesanal. Este autor formó parte de los arquitectos que discutieron a nivel regional lo "poco" latinoamericana de la arquitectura territarias 44 Especial 239 
${ }^{3}$ El Team X o Team 10, se refiere a un grupo de intelectuales de la arquitectura que se oponian al manifiesto de la Carta de Atenas proponiendo un ordenamiento urbano racionalista y moderno. Sus principales referentes eran Jaap Bakema, Georges Candilis, Giancarlo de Carlo, Aldo Van Eyck, Alison y Peter Smithson y Shadrac Woods.

\section{territorias 44 Especial}

subyacente del territorio. Por lo que desde la década del 60, junto a Fernando Salinas, autor de La arquitectura revolucionaria del Tercer Mundo (1970) y las discusiones en los Seminarios de Arquitectura Latinoamericana (SAL), se empezaron a exponer diversas corrientes de arquitectura.

Otros referentes que trabajan sobre una mirada crítica del quehacer arquitectónico en relación al medio y contexto son Enrico Tedeschi en Argentina (particularmente en Mendoza), primero desde la universidad en Buenos Aires, Tucumán, Córdoba y luego en Mendoza; y Marina Waisman, aún vigente por su legado al pensamiento latinoamericano, autora de La estructura histórica del entorno (1972) y Caracterización de una Arquitectura americana (1989), dos obras paradigmáticas que aportan al estudio de la historia de la arquitectura latinoamericana problematizada desde su contexto, entramado social y cultura importada de Europa y Norteamérica (colonización). Así mismo, el arquitecto Jorge Liernur (2001), es otro referente que se explaya sobre la historia del modernismo y el impacto en la arquitectura (división del trabajo y especialidades del hábitat) y en las formas de habitar. Se plantea la compleja relación que se dio al considerar la vivienda como mercancía en la que prima su valor de cambio.

Existen valiosos antecedentes académicos en las universidades públicas de Latinoamérica, de hecho, la mayoría de los autores citados ha realizado gran parte de sus aportes en las universidades públicas, en materias como historia, teoría, talleres de arquitectura, entre otras. En México y en Argentina, se exponen dos casos relacionados a las revueltas sociopolíticas de los 70. Por un lado, el Taller Total en la facultad de Arquitectura y Urbanismo de la Universidad Nacional de Córdoba, Argentina, de 1970 hasta 1976, y, por otro lado, Vivienda y Diseño Participativo (actualmente ADCP) de la corriente políticoacadémica denominada autogobierno a mediados de los años 70, en la facultad de Arquitectura de la UNAM, México.

El Taller Total surgió como contrapartida a los manifiestos expuestos por el Team X, ${ }^{3}$ que, desde 1953 comenzó a imponer las doctrinas sobre arquitectura y urbanismo en el Congreso Internacional de Arquitectura Moderna (C.I.A.M.). El arquitecto Eduardo Lastra (2015) cuenta la experiencia de este taller en la que se plantea una transformación de la relación universidad-sociedad-docente-estudiante; es decir, dada por los movimientos estudiantiles tanto como por los movimientos obreros. En este contexto de luchas sociales de diferentes sectores de la sociedad denominada "Cordobazo", se discute a la arquitectura como un fenómeno social de construcción colectiva con la sociedad, además de una respuesta técnica, se proponían ejes para la transformación de la universidad, el rol de los arquitectos y la vida estudiantil. Su legado es un antecedente valioso para la construcción del pensamiento crítico en la Argentina. 
En el caso mexicano, el autogobierno de la escuela de arquitectura surgió como una revuelta estudiantil que buscaba paliar la relación entre docentes-arquitectura empresarial. Como resultado se elaboró un Plan de Estudios de Autogobierno basado en 6 principios rectores: totalización de conocimientos, diálogo crítico, conocimiento de la realidad nacional, vinculación al pueblo y praxis. Dicha propuesta transformó la enseñanza de Arquitectura en la UNAM, promoviendo un antecedente inigualable en la Arquitectura latinoamericana. De esta experiencia se desprendió uno de los casos de estudio propuesto en este trabajo.

\section{Dos propuestas críticas $\mathrm{y}$ académicas de la Arquitectura}

Los dos casos expuestos en México y Argentina, son solo muestras del pensamiento crítico existente en el entramado de las redes académicas de la región, como la Red Universitaria Latinoamericana de Cátedras de Vivienda (ULACAV), la Coalición Internacional del Hábitat (HIC-LA) ${ }^{4}$ y el Encuentro Latinoamericano de Arquitectura Comunitaria (ELAC), entre otras.

Los casos propuestos son experiencias catedráticas de universidades públicas latinoamericanas, que abordan el problema del hábitat popular desde una episteme crítica y con diferentes métodos de abordaje.

En este apartado hay que destacar que en Latinoamérica se vienen aplicando métodos y experiencias novedosas respecto a las formas de intervención y renovación urbana de sectores deprimidos como es el urbanismo táctico (Lydon, 2012), urbanismo ciudadano (Verdaguer VianaCárdenas, 2002) o la acupuntura urbana (Lerner, 2005) por nombrar algunas. Si bien se reconoce la diversidad metodológica y la movilización de la participación en diferentes habitantes con la que se llevan a cabo estas iniciativas, no puede dejarse de lado la ausencia de integralidad tanto escalar como espacial de dichas propuestas que intervienen en reformas menores sin transformar los problemas urbanos estructurales. A su vez, no resulta más que una manera de patologizar los problemas urbanos y habitacionales, como refiere Galvis $(2020$, p. 3): "Las metáforas médicas en la planeación urbana han servido para replicar la marginalización y negación del derecho a la ciudad de determinadas poblaciones".

\section{La experiencia en México, el caso de la UNAM}

En México existen aproximadamente 110 escuelas de arquitectura en todo el país y alrededor de 22 están en la Ciudad de México. De acuerdo a una entrevista realizada a los docentes del Taller Max Cetto, ${ }^{5}$ egresan unos 3000 arquitectos por año en la ciudad capital, lo que constituye un total aproximado de 187000 profesionales ocupados, es decir, un/a arquitecto/a por cada 640 habitantes (Hernández Gálvez, 2014).
${ }^{4}$ La Coalición Internacional del Hábitat (HIC) Latinoamérica, quizás no ba incidido directamente en la formación y enseñanza de arquitectura, pero si ha influenciado por sus aportes a un pensamiento integrador del hábitat popular, la sistematización y construcción de red entre organizaciones sociales de base que responden y se constituyen en pos del derecho a habitar y por la basta bibliografia que ha publicado.

${ }^{5}$ Realizada el 16 de febrero del 2020.

\section{territarias 44 Especial} 241 


\section{tersitarios} 44 Especial
La línea de investigación Arquitectura, Diseño, Complejidad y Participación (ADCP) de la facultad de Arquitectura de la Universidad Nacional Autónoma de México, forma parte del campo del conocimiento del área de Análisis, Teoría e Historia (ATH) en el posgrado maestría y doctorado, y del Taller Max Cetto de la licenciatura o pregrado de Arquitectura. Tiene su origen en los movimientos académicos políticos de la década del 70 (movimiento estudiantil iniciado en 1966) denominado "Autogobierno" en la línea participativa y en las ONG Hábitat en México, promotoras de la producción, gestión social del hábitat y la vivienda y de la arquitectura y el diseño participativo. Parten de una integración del nivel de licenciatura y posgrados (especialización, maestría y doctorado), en el cual los estudiantes están relacionados en un trabajo conjunto, teórico, metodológico y de los ejercicios académicos. Se lleva a cabo una sistematización, difusión y reflexión con base en los seminarios organizados por los propios estudiantes con la participación de toda la línea. En la universidad y en la facultad se producen muchos trabajos, pero todos están dispersos y solo se difunde entre grupos cercanos, por ello, la línea pretende subsanar este problema a su escala y universo específico.

El horizonte epistemológico es crítico y se enmarca en las teorías de la complejidad. La Arquitectura se entiende como un término polisémico (por su variedad de comprensiones) que dialoga con multiciencias y saberes en su quehacer práctico. Principalmente la Arquitectura se aborda como ciencia mediadora de la construcción del espacio habitable en una relación dialógica como producto y producente. El espacio habitable se entiende entonces como el espacio en constante producción, configurado por la acción humana, más precisamente por las relaciones sociales de producción $y$, a su vez, establece una relación dialéctica compleja de la espacialidad con la vida humana. Se continúa así con lo expuesto por Henri Lefebvre (2013), quien promovió la idea de que el dominio sobre el espacio constituye una fuente fundamental y omnipresente del poder social sobre la vida cotidiana.

La línea ADCP parte de la premisa que el diseño participativo es el proceso con el cual se gesta el hábitat de producción social y vivienda (PSHV), concepto acuñado por organizaciones sociales en pos de definir formas colectivas y solidarias de construcción del espacio habitable. Se entiende que PSH "son todos aquellos procesos generadores de espacios habitables, que se realizan bajo el control de autoproductores y otros agentes sociales que operan sin fines de lucro [...]" (Ortiz, 2012, p. 74). Según Romero (2002), "el PSH apunta a que los distintos sectores puedan tener un hábitat que responda a sus demandas por medios de procesos en los que participen y decidan, entendiendo que el hábitat es un producto social en un proceso dialéctico" (p. 7). Los movimientos de pobladores y las organizaciones sociales de base 
luchan por garantizar un hábitat digno. Se entiende que un hábitat digno es la integralidad entre el acceso a los servicios básicos, vivienda y tierra, infraestructura y equipamiento básicos, los espacios de trabajo, producción y reproducción de la vida, el respeto por la diversidad, la cultura, la comunidad y el ambiente en el que vivimos (Miranda Gassull, 2017). Los procesos participativos permiten obtener las mejores posibilidades de producir condiciones urbano-arquitectónicas apropiadas, adecuadas con los recursos existentes que estimulan el desarrollo y potencial de los habitantes, y mejor entendimiento de las fuerzas y condiciones de la producción del hábitat para su continuo trabajo en transformación.

La frase publicada por Romero et al. (2018): "Los arquitectos no inventaron la PSH sino que la encontraron en los barrios [...]", es una afirmación que nos introduce en el complejo universo epistemológico que tiene la arquitectura en su giro crítico y deconstructivista del modernismo y de las realidades (y sujetos) latinoamericanos. En ese sentido, hay que destacar que desde los años 70 se promueve una producción académica regional en Latinoamérica sobre estas prácticas y su impacto en la formación de Arquitectura. La línea ADCP es una de las principales referentes del continente, es un equipo que promueve la participación como una postura de poder (hacer y saber) como un posicionamiento ideológico.

La incorporación de otros sujetos, no es en realidad una integración de los otros, sino más bien, una deconstrucción de la individualidad propia de la Arquitectura y a los sectores apunta. Nadie se animaría a decir que alguien no merece acceder a la arquitectura, sin embargo, en el discurso científico con el que se imparte el proceso proyectual, teórico y metodológico de esta, se quedan varios sectores en el camino. La propuesta del ADCP (véase figura 1), parte de la premisa que la construcción del espacio habitable no es otra cosa que la continua relación democrática y recíproca entre los habitantes y quienes participan como trabajadores de la construcción, es un proceso dialéctico y dialógico en el cual se construyen relaciones de poder hacer y saber.

La afectividad es un tema central para abordar la participación desde los sujetos que protagonizan un proceso, hacer hincapié en el sentimiento de arraigo, de pertenencia a gusto y a escala humana. El concepto de micrópolis también construye la relevancia de las personas que intervienen en un proceso de diseño participativo, ya que la escala local, micro, pone de relevancia otro lugar de los cuerpos-sujetos-casas-barrio involucrados.

La estrategia de toma del diseño se basa en el concepto de "micrópolis" como una escala posible de abordar el tejido social de un determinado fenómeno o problema de la ciudad existente, por ejemplo, un barrio es la célula del tejido social por excelencia en las ciudades. La micrópolis son cortes y rupturas de la ciudad, de un fenómeno de mayor escala que se recortan territarias 44 Especial 243 
para poder comprender su dinámica. Esto no quiere decir que se desvincula de una escala mayor, y mucho menos de una escala menor, sino que se utiliza una escala de micrópolis como herramienta de análisis para el reconocimiento urbano tanto de las problemáticas como de los actores, sus sentires, sus funciones, en un determinado espacio. En cuanto al crecimiento, se propone con la idea de "barrio participativo, sustentable y con visión de género" que permite abordar un proceso continuo integral, articulado con las decisiones de los diferentes actores involucrados.

La construcción del espacio habitable se enseña promoviendo dos atributos centrales para abordar las necesidades sociales de habitar. En primer lugar se incentiva la actitud como la capacidad de entender la complejidad del hábitat y la pobreza urbana. En segundo lugar se propone estimular la aptitud como la capacidad de hacer, concretar y llevar a la praxis aquello que se ha construido a partir del entendimiento. La participación no es solo una metodología o una forma de construcción de poder (política), sino es una manera de comprender al mundo.

A partir de una investigación realizada por el grupo de HABYTED-CYTED publicada en tres libros, al respecto se encontró que, en primera instancia, en el caso de tersitorias 44 Especial 244
Figura 1. Conceptos clave de la línea ADCP

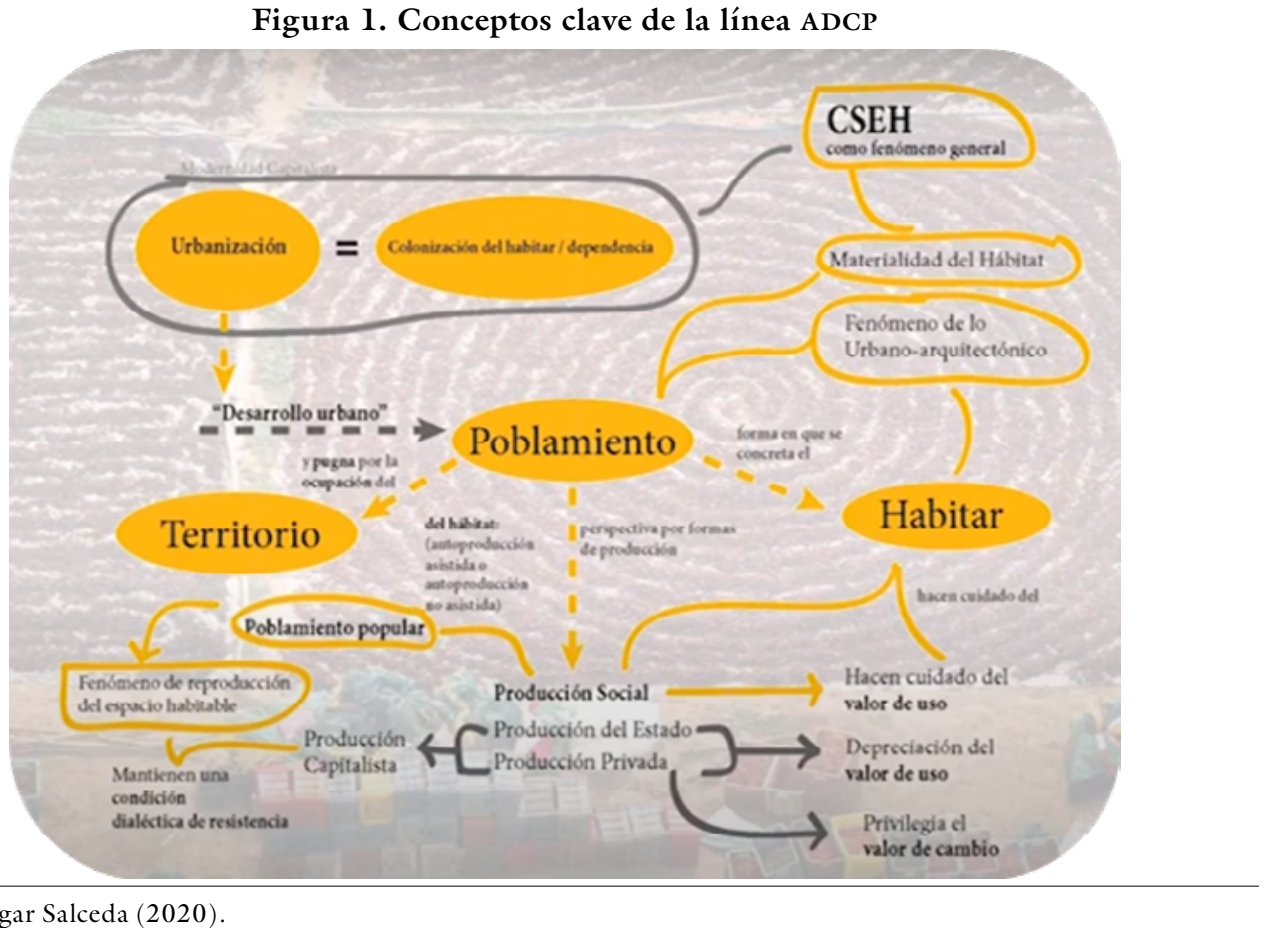

VIRGINIA MiRANDA GASSULL 
América Latina existían solo cuatro propuestas de diseño participativo que tenían teoría, metodologías y prácticas que son las que a continuación se enuncian. Los principales autores que se toman para conformar la batería de métodos de enseñanza son Livingston (arquitectos de familia), Christopher Alexander, Sarah Ishikawa y Murray Silverstein (lenguaje de patrones), John Habraken (teoría de los soportes) y Michael Pyatok y Hanno Weber (teoría de diseño participativo).

$\mathrm{El}$ arquitecto Alexander es un referente contemporáneo que aporta métodos comprobados para practicar la utopía de la arquitectura y el urbanismo producto de la participación política comunitaria. Este autor parte de la idea que el diseño habitacional es un problema complejo, que necesita de diversos actores para pensarse en conjunto. Propone una mirada sistémica y sistematizada de posibles soluciones partiendo de la situación real y el mundo imaginado, para concluir soluciones sometidas a juicios de todos los participantes. Este arquitecto tiene como antecedente a su legado del pensamiento, un trabajo realizado en Perú, presentado en el concurso PREVI en 1970, en el que la realidad del lugar, la cultura migratoria del sector y su particular forma de resolver lo habitacional, despertaron su comprensión de un mundo por fuera de la racionalización moderna.

Por su lado, John Habraken, fue un arquitecto holandés que incidió en pleno auge e implantación del modernismo urbano en Europa. Su propuesta radica en visibilizar lo más importante del problema habitacional, sus habitantes y deseos/ necesidades. En correlación, su aporte se basa en distinguir el soporte (terreno fijo con áreas comunes) del relleno (obra) y elementos movibles. Hay que aclarar que soporte no refiere a la estructura o reticulado. Como expresa Colmenares:

El concepto de soporte nace de la distinción entre la prefabricación de las partes y la repetición del conjunto y constituye el primer paso hacia la definición de un sistema abierto en el que la vivienda colectiva pueda llegar a conformarse de forma específica [...]. El mejor soporte es el que sugiere al individuo formas de ser ocupado (2010, p. 5).

El mejor soporte no es el más flexible si no el que más se adecúa al grupo social al que se dirige (Romero \& Mesías, 2004). La obra final pierde valor desde esta teoría, importa el proceso, el consenso y la capacidad evolutiva de llevar a cabo una propuesta en común.

Michael Pyatok y Hanno Weber discuten desde sus inicios las formas en las que se imparte el diseño como un asunto privado y movilizado por juicios estéticos modelados en valores estéticos, normalizadores y de supuesta neutralidad de los profesionales. Los autores promulgan una posición más política al abordaje del hábitat popular y el diseño participativo, discutiendo las bases epistemológicas que territarios 44 Especial 245 
fundaron el movimiento moderno proponiendo una relación dialéctica entre la arquitectura y la sociedad. Como afirma Romero et al. (2018):

Así, desarrollan un procedimiento consistente en la generación de opciones originadas por el intercambio igualitario de actores y arquitectos, cuya comunicación da lugar a la representación gráfica de posibles soluciones decididas por la comunidad de destinatarios, con el apoyo del arquitecto como gestor, negociador y aportador de saberes específicos (p. 131).

El aporte es una discusión rigurosa y científica sobre el a priori positivista y la neutralidad que se ejerce en la formación de Arquitectura, que luego son reproducidos por los profesionales en su quehacer práctico. Es quizás la propuesta que permite trabajar más claramente con el conjunto de actores involucrados: los habitantes, los vecinos, las autoridades, los profesionales, entre otros, en todo el proceso de decisiones que va desde las concepciones más abstractas hasta las más concretas.

Hay dos autores latinoamericanos que se han vinculado a los referentes nombrados anteriormente, han tenido una larga trayectoria de experiencias tanto en gestión habitacional como en la vida académica sobre el diseño participativo y la producción social del hábitat. Estos autores son Mariana Enet (Córdoba, Argentina) y Gustavo Romero (UNAM), quienes trabajan colectivamente en más de una ocasión.

Mariana Enet ha desarrollado diversas experiencias de diseño participativo en la región, que le han valido de un sistema espiralado (diagnóstico, planeación, monitoreo, evaluación y comunicación) para modelar las diferentes etapas/ritmos de un proceso de diseño participativo. Este sistema resulta un lenguaje posible para llevar a cabo prácticas concretas y efectivas con diferentes comunidades en territorios diversos.

El arquitecto Romero, por su parte, ha sido uno de los constructores de la relación académica y la PSHV en México, a partir de resignificar a los autores nombrados anteriormente en la praxis docente y constituir su propio bagaje conceptual y metodológico. Romero ha sido partícipe de diferentes experiencias que han gestado antecedentes de diseño participativo en diversas comunidades con resultados prominentes en la región, lo que significa un legado más que significativo en nuestros territorios y una prueba irrefutable de que debe incorporarse en los currículos de Arquitectura este campo de estudio y acción.

La línea ADCP es una propuesta epistemológica que responde a la necesidad de pensar otra arquitectura posible, que revive el legado de las revueltas del "autogobierno" a partir de continuar marcando una posición crítica y posible de discutir lo existente. A modo de cierre, Romero y Mesías culminan con que "la base para 
una nueva manera de concebir el diseño se encuentra una forma diferente de conocer, entender y explicar la realidad, es decir una nueva epistemología” (2004b, p. 59).

\section{La experiencia en Argentina, el caso de la UNCUYO}

En Argentina, el Consejo Interuniversitario Nacional (CIN) afirma que existen altos niveles de pobreza y de población socialmente excluida, por lo que la necesidad de producir cambios socioeconómicos, reconstruir los lazos sociales y garantizar la justicia social e igualdad de oportunidades, constituyen una dimensión fundamental que debe incidir en la definición de políticas educativas, científicas y tecnológicas para los próximos años (CIN, 2004). En el país existen más de cincuenta instituciones educativas del ámbito público y privado que ofertan la carrera de Arquitectura y aproximadamente unos 61000 profesionales egresados con título de arquitectos. Sin embargo, según la Federación Argentina de Entidades de Arquitectos (FADEA) y la Sociedad Central de Arquitectos (SCA) desde el 2013 aumentó considerablemente el número de estudiantes de Arquitectura y, con ello, el número de egresados; se estima que hay 1,46 profesionales (habilitados $)^{6}$ por cada 1000 habitantes en la Argentina (Baldo, 2013). Este número alarma a las instituciones que regulan la profesión de la Arquitectura, ya que el número de profesionales por habitante es muy elevado, lo que provoca una depreciación en el empleo.

La situación habitacional deficiente en Argentina es del $28 \%$, que representa una necesidad total de 3000000 millones de vivienda nueva o de acondicionamiento. Dicha situación muestra que existe un alto porcentaje de la población que necesita de profesionales arquitectos involucrados en los procesos de producción habitacional de los sectores populares o de bajos ingresos de la sociedad.

¿Qué grado de importancia tiene la problemática habitacional de los sectores populares en la formación universitaria de la Arquitectura? Para revelar el interrogante, se realizó una matriz descriptiva y comparativa de la presencia de las cátedras referidas a la problemática del hábitat solo en instituciones públicas nacionales. El diseño metodológico utiliza fuentes primarias y secundarias referidas a los planes de estudios de las universidades públicas. El análisis documental se realizó a través de la comparación de las carreras de Arquitectura de cada universidad en relación a las siguientes variables: áreas temáticas de cada carrera, carácter obligatorio u optativo de las materias referidas a la temática de hábitat o vivienda social, contenido de los programas de cada cátedra, carga horaria y año académico en el que se imparte la materia.

Mediante el análisis desarrollado en tabla 1 , se puede observar que en la mayoría de las carreras el tema de hábitat popular no tiene una jerarquía destacada,
${ }^{6}$ Solo la mitad de los egresados de Arquitectura del pais se matriculan para habilitar el ejercicio profesional. territarios 44 Especial 247 
Tabla 1. Cuadro comparativo entre carreras de Arquitectura de universidades públicas

\begin{tabular}{|c|c|c|c|c|}
\hline n. ${ }^{\circ}$ & Universidad & Vivienda Social-Comunidad & Año & Tiempo \\
\hline \multirow{10}{*}{1} & \multirow{10}{*}{$\begin{array}{l}\text { Universidad } \\
\text { Arquitectura Buenos } \\
\text { Aires }\end{array}$} & $\begin{array}{l}\text { Curso interdisciplinario para la } \\
\text { urgencia social }\end{array}$ & \multirow{3}{*}{$\begin{array}{l}\text { Optativa a partir } \\
\text { de } 4 .^{\circ} \text { año }\end{array}$} & \multirow{3}{*}{$\begin{array}{l}1 \text { semestre, } \\
60 \mathrm{~h}\end{array}$} \\
\hline & & $\begin{array}{l}\text { El arquitecto en la gestión pública y } \\
\text { social }\end{array}$ & & \\
\hline & & Diseño del hábitat accesible & & \\
\hline & & $\begin{array}{l}\text { La arquitectura en la producción } \\
\text { urbana latinoamericana }\end{array}$ & Optativa & $120 \mathrm{~h}$ \\
\hline & & Gestión urbana contemporánea & Optativa & $\begin{array}{l}\text { Cuatrimestral, } \\
52 \mathrm{~h}\end{array}$ \\
\hline & & $\begin{array}{l}\text { Proyecto, participación y habitar } \\
\text { (Proyecto Habitar) }\end{array}$ & & \\
\hline & & Programa de hábitat inclusivo & & \\
\hline & & $\begin{array}{l}\text { Taller libre de proyecto social. Área de } \\
\text { hábitat }\end{array}$ & $\begin{array}{l}\text { Cátedra } \\
\text { interdisciplinaria }\end{array}$ & Semestral, $60 \mathrm{~h}$ \\
\hline & & \multicolumn{3}{|c|}{ Estudios urbanos y de la vivienda en América Latina (MEUVAL) } \\
\hline & & \multicolumn{3}{|c|}{ Maestría Hábitat y Pobreza Urbana en América Latina (MHyPUAL) } \\
\hline \multirow{2}{*}{2} & \multirow{2}{*}{$\begin{array}{l}\text { Universidad de San } \\
\text { Martín }\end{array}$} & Taller de gestión urbana & $5 .^{\circ}$ año & Semestral, $64 \mathrm{~h}$ \\
\hline & & \multicolumn{3}{|c|}{ Posgrado en Hábitat Social en Territorio Local } \\
\hline \multirow{2}{*}{3} & \multirow{2}{*}{$\begin{array}{l}\text { Universidad Nacional } \\
\text { Tres de Febrero }\end{array}$} & \multicolumn{3}{|l|}{ Maestría en Políticas Sociales Urbanas } \\
\hline & & \multicolumn{3}{|c|}{ Seminario de posgrado: Políticas de Hábitat y Mercados } \\
\hline \multirow[t]{2}{*}{4} & \multirow[t]{2}{*}{$\begin{array}{l}\text { Universidad Nacional } \\
\text { de Mar del Plata }\end{array}$} & $\begin{array}{l}\text { Derecho a la ciudad y producción del } \\
\text { espacio. Tensiones y contradicciones } \\
\text { con la Nueva Agenda Urbana }\end{array}$ & Optativa & $\begin{array}{l}\text { Cuatrimestre, } \\
45 \mathrm{~h}\end{array}$ \\
\hline & & \multicolumn{3}{|l|}{ Maestría en Hábitat y Vivienda } \\
\hline \multirow{3}{*}{5} & \multirow{3}{*}{$\begin{array}{l}\text { Universidad Nacional } \\
\text { de Córdoba }\end{array}$} & $\begin{array}{l}\text { Problemática de la vivienda popular } \\
\text { (I y II) }\end{array}$ & $\begin{array}{l}\text { Optativa a partir } \\
\text { de } 4 .^{\circ} \text { año }\end{array}$ & $\begin{array}{l}\text { Semestral, } \\
37,5 \mathrm{~h}\end{array}$ \\
\hline & & $\begin{array}{l}\text { Práctica profesional asistida con } \\
\text { orientación en hábitat popular }\end{array}$ & Obligatoria & Semestral, $60 \mathrm{~h}$ \\
\hline & & Taller 36 & Optativa & \\
\hline
\end{tabular}




\begin{tabular}{|c|c|c|c|c|}
\hline n. ${ }^{\circ}$ & Universidad & Vivienda Social-Comunidad & Año & Tiempo \\
\hline & & $\begin{array}{l}\text { Seminario de gestión territorial y } \\
\text { hábitat }\end{array}$ & Optativa & $32 \mathrm{~h}$ \\
\hline & & Diseño cooperativo & $\begin{array}{l}\text { Optativa a partir } \\
\text { de } 4 .^{\circ} \text { año }\end{array}$ & $\begin{array}{l}1 \text { cuatrimestre, } \\
40 \mathrm{~h}\end{array}$ \\
\hline & & \multicolumn{3}{|c|}{ Maestría en Gestión y Desarrollo Habitacional } \\
\hline \multirow{4}{*}{6} & \multirow{4}{*}{$\begin{array}{l}\text { Universidad Nacional } \\
\text { de Rosario }\end{array}$} & $\begin{array}{l}\text { Arquitectura, comunidad y territorio. } \\
\text { Introducción a la extensión } \\
\text { universitaria }\end{array}$ & \multirow{3}{*}{$\begin{array}{l}\text { Optativa a partir } \\
\text { de } 3 .^{\circ} \text { año }\end{array}$} & \multirow{3}{*}{$\begin{array}{l}1 \text { semestre, } \\
30 \mathrm{~h}\end{array}$} \\
\hline & & $\begin{array}{l}\text { Del proyecto y la gestión de la vivienda } \\
\text { social. Aspectos multidimensionales del } \\
\text { derecho al hábitat }\end{array}$ & & \\
\hline & & $\begin{array}{l}\text { Todos para una. Una para todas. } \\
\text { Inclusividad arquitectónica }\end{array}$ & & \\
\hline & & \multicolumn{3}{|c|}{ Especialización en Gestión Local del Hábitat (FAPYD) } \\
\hline 7 & $\begin{array}{l}\text { Universidad } \\
\text { Tecnológica Nacional- } \\
\text { Facultad Regional } \\
\text { Santa Fe }\end{array}$ & $\begin{array}{l}\text { El hábitat desde una perspectiva } \\
\text { interdisciplinaria }\end{array}$ & Optativa & $90 \mathrm{~h}$ \\
\hline 8 & $\begin{array}{l}\text { Universidad Nacional } \\
\text { de la Plata }\end{array}$ & $\begin{array}{l}\text { Hábitat popular: problemas, políticas y } \\
\text { gestión }\end{array}$ & Optativa & $\begin{array}{l}1 \text { semestre, } \\
45 \mathrm{~h}\end{array}$ \\
\hline \multirow{3}{*}{9} & \multirow{3}{*}{$\begin{array}{l}\text { Universidad Nacional } \\
\text { del Nordeste }\end{array}$} & $\begin{array}{l}\text { Imaginarios urbanos: participación } \\
\text { social, planificación y gestión } \\
\text { urbanísticas }\end{array}$ & Optativa & Semestral \\
\hline & & $\begin{array}{l}\text { Derechos humanos y participación } \\
\text { ciudadana }\end{array}$ & Cátedra libre & Semestral, $40 \mathrm{~h}$ \\
\hline & & $\begin{array}{l}\text { Gestión y desarrollo de la vivienda } \\
\text { popular }\end{array}$ & $\begin{array}{l}\text { Obligatoria en } \\
5 .^{\circ} \text { año }\end{array}$ & Semestral, $40 \mathrm{~h}$ \\
\hline 10 & $\begin{array}{l}\text { Universidad de } \\
\text { Concepción del } \\
\text { Uruguay }\end{array}$ & \multicolumn{3}{|l|}{ No existe } \\
\hline \multirow{2}{*}{11} & \multirow{2}{*}{$\begin{array}{l}\text { Universidad Nacional } \\
\text { de Avellaneda }\end{array}$} & Trabajo social comunitario I & $\begin{array}{l}\text { Obligatoria en } \\
1 .^{\circ} \text { año }\end{array}$ & Semestral, $32 \mathrm{~h}$ \\
\hline & & Trabajo social comunitario II & $\begin{array}{l}\text { Obligatoria en } \\
\text { 2. } \text { `año }^{\text {año }}\end{array}$ & Semestral, $32 \mathrm{~h}$ \\
\hline
\end{tabular}




\begin{tabular}{|c|c|c|c|c|}
\hline n. ${ }^{\circ}$ & Universidad & Vivienda Social-Comunidad & Año & Tiempo \\
\hline & & Trabajo social comunitario II & $\begin{array}{l}\text { Obligatoria en } \\
3 .^{\circ} \text { año }\end{array}$ & Semestral, $32 \mathrm{~h}$ \\
\hline & & Trabajo social comunitario II & $\begin{array}{l}\text { Obligatoria en } \\
4 .^{\circ} \text { año }\end{array}$ & Semestral, $32 \mathrm{~h}$ \\
\hline & & Historia de la vivienda social & Optativa & $32 \mathrm{~h}$ \\
\hline & & Hábitat popular & $\begin{array}{l}\text { Optativa a partir } \\
\text { de } 6 .^{\circ} \text { año }\end{array}$ & Semestral, $50 \mathrm{~h}$ \\
\hline \multirow[b]{2}{*}{12} & \multirow{2}{*}{$\begin{array}{l}\text { Universidad Nacional } \\
\text { de San Juan }\end{array}$} & \multirow{2}{*}{ Vivienda de interés social } & $4 .^{\circ}$ año & \multirow{2}{*}{ Semestral } \\
\hline & & & Optativa & \\
\hline 13 & $\begin{array}{l}\text { Universidad Nacional } \\
\text { del Litoral }\end{array}$ & Vivienda social y ciudad & Optativa & Semestral, $45 \mathrm{~h}$ \\
\hline 14 & $\begin{array}{l}\text { Universidad Nacional } \\
\text { de Tucumán }\end{array}$ & Arquitectura en tierra cruda & Optativa & Semestral, $48 \mathrm{~h}$ \\
\hline 15 & $\begin{array}{l}\text { Universidad Nacional } \\
\text { de Cuyo }\end{array}$ & Vivienda de interés social & Obligatoria & Semestral, $45 \mathrm{~h}$ \\
\hline
\end{tabular}

Fuente: elaboración propia (2020).

prevalece la vivienda social o vivienda de interés social como denominación de las cátedras que abordan el tema. La minoría de las carreras analizadas incorpora en los currículos obligatorios el curso de al menos una materia que se relacione con la problemática habitacional. El resto de las universidades propone cátedras de carácter optativo, quedando a expensas de cada estudiante el contacto con el mundo del hábitat popular. Sin embargo, es preciso destacar que el problema no solo se sustenta en si es optativa u obligatoria, sino en el carácter subyacente que se le otorga. Hay que destacar que existen otros espacios académicos en las facultades de
Arquitectura que abordan el tema del hábitat popular, como centros de investigación, posgrados (que se incluyen en la tabla) que ofertan y producen conocimiento local.

A partir del 2006 se produjeron cambios sustantivos en tres carreras de Arquitectura del país, basados en la incorporación de la temática del hábitat popular a los currículos obligatorios de los planes de estudio. Se considera que dicha fecha está estrechamente vinculada a la crisis económica, social y política que atravesó la Argentina en el 2001, en la que se empobreció un alto porcentaje de la población, y con ello, la necesidad habitacional se volvió una problemática a 
la orden del día, que aún en la actualidad no se puede resolver totalmente en el país. A partir del 2001, los sectores populares se organizaron en torno a figuras colectivas para acceder a soluciones habitacionales, modelo que fue impulsado a partir del 2004 con el gobierno oficial basado en el Plan Federal de Viviendas que contenía el programa para jefa y jefes de hogar (desempleados), dirigido a promocionar trabajo relacionado con la construcción.

Se toma como caso de estudio argentino a la Universidad Nacional de Cuyo (si bien tiene larga data de creada, fundada en 1939), recién desde el 2011 se creó la joven carrera de Arquitectura en la Argentina en la facultad de Ingeniería. La misma tiene una estructura programática destacada por la presencia obligatoria de la cátedra de Vivienda de Interés Social (cátedra VIS). La incorporación de la problemática habitacional la pone a la vanguardia en la educación superior universitaria, promoviendo profesionales que sean reflexivos sobre la realidad sociohabitacional en las que están insertas y en donde se desarrollará su práctica profesional. Uno de los objetivos principales de la carrera es la concepción de la arquitectura como ciencia (básica, tecnológica y social) y como arte en la formación de profesionales capaces de dar respuestas sustentables a los diversos contextos (geográficos, culturales, sociales, económicos y políticos). No obstante, el obstáculo epistemológico del abordaje sobre el hábitat popular se da por la limitada transversalidad que existe entre otros espacios académicos del trayecto obligatorio, que reconozcan a otros usuarios/habitantes a los que la arquitectura debiera responder, como también, el desconocimiento sobre las corrientes críticas y revisionistas de la Arquitectura.

La cátedra se posiciona en los aportes de la educación popular de Paulo Freire, y más precisamente, en el campo de la extensión crítica latinoamericana que parte de la educación popular, la resignifica en el contexto actual desde una construcción dialógica entre el conocimiento universitario y las necesidades sociales.

La cátedra de VIS propone como primer paso el encuentro con el otro y la otra, es decir, el reconocimiento de la diversidad, la alteridad y necesidad de construcción de puntos en común. La primera aproximación con los estudiantes refiere a repensarse como sujetos parte del problema habitacional, tanto usuarios como técnicos, seres políticos, culturales y sociales. Esto quiere decir, poner el centro del problema como algo propio y parte de la realidad que vivimos, donde los arquitectos y las arquitectas son mediadores, a la vez que habitantes. Esta relación obvia deconstruye (o al menos lo intenta) la racionalización y objetivación de la arquitectura y sus actores.

Si bien la materia se denomina $\mathrm{Vi}^{-}$ vienda de Interés Social, en el constructo teórico se parte de concebir el problema de la vivienda como un tema integral del hábitat. El término hábitat se destaca como territarios 44 Especial 


\section{territorias} 44 Especial una concepción superadora de la vivienda, configurado como el espacio que se funda materialmente a partir de la construcción de vivienda, equipamiento, conectividad, suelo, relaciones económicas y obras de infraestructura; y simbólicamente por las relaciones sociales, figuradas (estatus) y corpóreas. El hábitat está fijado a un determinado territorio (el Estado establece sus límites administrativos-políticos) regido por normativas, legislaciones y decisiones políticas que influencian el cómo se produce la vivienda, y por lo tanto, condiciona la configuración material del hábitat. La vivienda es un satisfactor y no la necesidad, la necesidad es de habitar. El hábitat además está determinado socialmente a partir de la interacción entre los sujetos y grupos sociales que habitan un mismo territorio y donde se producen sus prácticas sociales y productivas cotidianas (Miranda Gassull, 2017).

El abordaje de la problemática habitacional como un tema estructural $-\mathrm{y}$ no basado en la cantidad de viviendas faltantes- de la sociedad, establece el vínculo entre los procesos históricos, decisiones políticas, económicas y el medio social en el que se establecen las relaciones de fuerzas que se reproducen en el contexto del estudiante y futuro profesional. Al pensar la vivienda desde una concepción amplia, compleja e integradora como es el concepto de hábitat, se puede discutir e introducir la pluralidad disciplinaria que coexiste en el abordaje de soluciones habitacionales y ampliar el campo académico de la arquitectura.

Un primer paso para ampliar el campo de la arquitectura que renueva a su vez el rol profesional, es abordar al hábitat humano desde la transformación del concepto de vivienda terminada, en pos de una vivienda en proceso. El arquitecto autor ya no es el eje de la respuesta habitacional, sino que por el contrario, es un actor más del proceso habitacional - puede ser a través del acompañamiento técnico en organizaciones sociales autogestionarias y en el diseño, implementación y programación de políticas públicas habitacionales-. En ese marco, se adhiere a lo expuesto por línea ADCP e HIC-LA en México sobre los estudios valiosos de las experiencias de la producción social del hábitat (PSHV) en Latinoamérica y la necesaria articulación que debe existir entre el campo científico y el político-técnico.

Entonces, se comprende el hábitat como producto de las relaciones humanas y no como un espacio limitado determinado por su configuración. Para establecer qué entendemos por hábitat, es necesario definir quiénes son sus habitantes, cómo son sus redes sociales, sus condiciones físicas, culturales, económicas y simbólicas (véase figura 2). Además, es necesario contextualizar su historia, sus saberes y su espacialización. Resulta importante señalar lo que expresan Álvarez y Blanco (2013, p. 9) sobre el habitar desde un sentido colectivo retomando los aportes de Deleuze (1987) sobre la subjetividad 
como una producción colectiva que surge del entramado relacional y el contexto socio-histórico en el pliegue del "afuera" que conforma el "adentro". Es decir, habitar es siempre colectivo fundado en la trama de la vida. Así bien, tanto habitar en la intimidad como en la vida pública está atravesado por el sentido colectivo, relacional y vincular; la diferenciación escalar se establece como un orden para organizar los contenidos impartidos.

La práctica de la materia se realiza desde un abordaje que desarrolla el aprendizaje basado en la investigación y acción participativa que promueve un espíritu crítico y reflexivo. El campo de la extensión del aula taller en el territorio es la premisa fundamental del abordaje de la materia (véase figura 3). Se afirma así lo expuesto por la Universidad de la República del Uruguay (referente en la temática a nivel regional): "La extensión y la investigación deberían ser parte de la metodología de enseñanza universitaria, lo que haría que el proceso formativo fuese integral, con un contacto directo con la realidad social, por lo tanto humanizadora" (UDELAR, 2009, pp. 3-4).

El diseño metodológico y las técnicas empleadas de diverso origen disciplinario y experiencial conforman un fecundo cuerpo instrumental para los procesos

Figura 2. El habitar como acto-relación con el movimiento

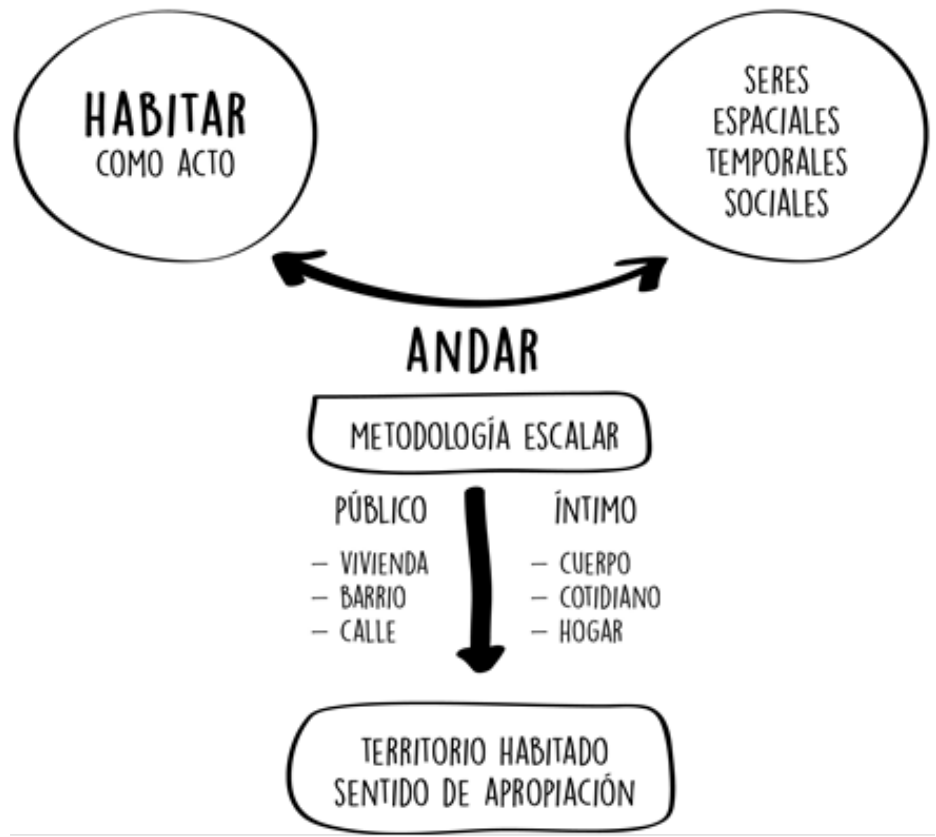

Fuente: elaboración propia (2016). 
de enseñanza, planificación participativa, sistematización de experiencias, entre otras acciones. La concepción "metodológicadialéctica" (Jara, 2017) refiere a una distinción entre las técnicas empleadas y el procedimiento metodológico a utilizar. De modo tal que la teoría y la metodología empleada sea coherente, dialógica y factible de realizar en el tiempo calendario de la materia (un semestre de 45 horas), se propone como objetivo principal que, a través del ejercicio práctico de la gestión habitacional de un caso real de estudio, identificar a los actores protagonistas del proceso habitacional, comprender las disciplinas involucradas, las etapas de desarrollo y las diferentes escalas que involucran una solución de vivienda de interés social.

El procedimiento metodológico empleado sobre la propuesta práctica refiere a dos modalidades: el aula taller y trabajo en el territorio (extensión participativa).

Figura 3. Procedimiento metodológico de la cátedra vis

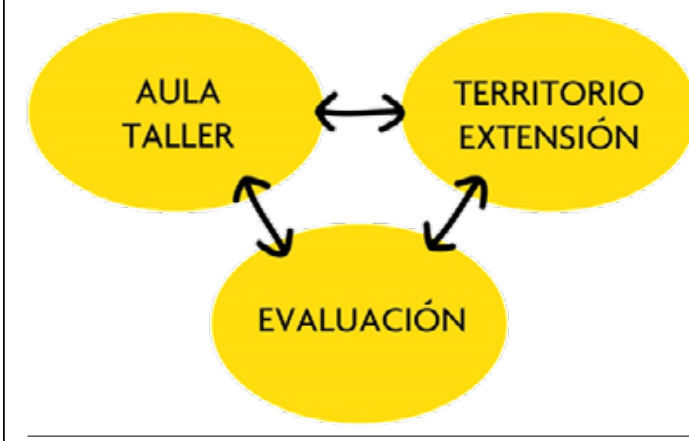

Fuente: elaboración propia (2016).
El trabajo del aula taller (véase figura 4) busca dinamizar descubrimientos mediante un proceso colectivo de diagnóstico, problematización y diseño de una propuesta arquitectónica. En el aula se imparte el conocimiento teórico planteado en tres unidades que abarcan el conocimiento teórico teniendo como ejes principales: la diversidad de sujetos (1), el hábitat como objeto multidimensional (2) y los territorios (3). El abordaje teórico y la contextualización histórica del problema habitacional son fundamentales para lograr la conciencia del problema estructural del acceso a la vivienda, el suelo, el trabajo y un ambiente saludable.

\section{Conclusión}

El rol del Estado como garante del derecho a habitar es imprescindible para iniciar un proceso de trasformación en las formas de acceso a un hábitat digno y, es en este sentido que la universidad cumple un rol fundamental como actor de dicho Estado. Por lo tanto, la formación académica en Arquitectura se estructura con un formato que va "detrás" de los problemas sociales, que no anticipa en el grado universitario futuros profesionales capaces de insertarse en sus contextos inmediatos, que dispongan de la capacidad técnica, artística y creativa hacia toda la sociedad.

Este trabajo presenta los diversos autores y metodologías utilizadas para pensar la arquitectura desde una mirada crítica, pero aún más relevante resulta desentrañar 
Figura 4. Organización del proceso metodológico

\begin{tabular}{|c|c|c|c|}
\hline & \multicolumn{3}{|c|}{ AULA TALLER } \\
\hline 7 & & aso de estudio & \\
\hline Z & 1ra Etapa & 2da Etapa & 3ra Etapa \\
\hline 虍 & $\begin{array}{l}\text { De la Necesidad } \\
\text { al Problema. }\end{array}$ & $\begin{array}{l}\text { Del Análisis al } \\
\text { Diagnóstico }\end{array}$ & $\begin{array}{l}\text { Del Diagnóstico a } \\
\text { la Programación }\end{array}$ \\
\hline 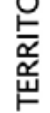 & Planteo problemático & $\begin{array}{l}\text { Diagnóstico y } \\
\text { Pre-programa de } \\
\text { necesidades }\end{array}$ & $\begin{array}{l}\text { Propuesta de diseño } \\
\text { Habitacional }\end{array}$ \\
\hline
\end{tabular}

Fuente: elaboración propia (2016).

los principios dominantes de la modernidad que tanto mal han hecho a nuestra región. En ese marco, se cuestiona el conocimiento eurocéntrico y anglosajón que copó a las escuelas de Arquitectura en Latinoamérica, modelando las formas, las ideas y las ciudades de nuestros territorios. Sin embargo, el gran aporte no está en esta discusión - de la que ya existe una gran cantidad de bibliografía-, sino que radica en desentrañar nuestra propia modernidad, es decir, esa mutación moderna que volvimos local, que se ha reproducido desde nuestros saberes, conocimientos y prácticas como nativos de esta región, desgarrando la propia realidad. La masiva producción social del hábitat que existe en todos los territorios latinoamericanos, da cuenta de otras formas posibles de construcción social del espacio habitable, son la viva crítica al mundo modernizante y su cara más cruda. Las experiencias aquí expuestas, como muchas otras existentes, y que a su vez trabajan en red, representan una vertiente del pensamiento latinoamericano crítico del hábitat humano, que ponen a la arquitectura en acción pensándola y configurándola desde nuestras realidades y territorios.

Lo curioso de los dos casos expuestos es que si bien las facultades de Arquitectura distan de una diferencia temporaria de más de 100 años y de muchos kilómetros que marcan contextos totalmente disímiles, el lugar que ocupa la perspectiva crítica en la enseñanza de Arquitectura resulta similar en ambos lugares y se reduce a un grupo de académicos o activistas sociales que la promueven.

A modo de conclusión se puede exponer entonces que, la necesaria construcción del pensamiento crítico en Latinoamérica sitúa en un lugar de importancia el rol de las facultades de Arquitectura en la transformación del pensamiento racionalista y mercantil que impera en la práctica profesional. La incorporación de la diversidad de la cultura latinoamericana en la formación tersitarios 44 Especial 255 
arquitectónica es aún un tema que se debe transversalizar en los currículos. Las propuestas académicas en este artículo no son completas ni universales, pero sí evidencian un posicionamiento crítico en la enseñanza de Arquitectura, que apela a su vez a la construcción de experiencias académicas que propongan revertir la relación universidad-sociedad dominante, en la que se anteponga la sociedad como objetivo principal de la universidad.

\section{Referencias}

Álvarez, E., \& Blanco, V. (2013). Componer, habitar, subjetivar. Aportes para la etnografía del habitar. Bifurcaciones. Revista de estudios culturales urbanos, $9(15)$. http://www.bifurcaciones.cl/2013/12/componer-habitarsubjetivar/

Baldo, P. (2013, 22 de noviembre). ¿Hay más arquitectos de los necesarios? Diario Clarin. https://www.clarin.com/ arq/arquitectura/arquitectosnecesarios_0_HkBfPQQiD7x.html

Barreto, M. (2006). La interdisciplina en el abordaje académico del hábitat social "informal": Fundamentos, líneas de acción y obstáculos a partir de la carrera de arquitectura. Revista INVI, 21(56), 16-30. http://revistainvi. uchile.cl/index.php/INVI/article/ view/306/895

tersitorios 44 Especial
Carlevaro, P. (2010). Intersecciones y uniones de la universidad con la ética.
Reencuentro. Análisis de problemas universitarios, (57), 8-17. https://reencuentro.xoc.uam.mx/index.php/ reencuentro/article/view/738

Castaño, J., Bernal, M., Cardona, D., \& Ramírez, I. (2005). La enseñanza de la arquitectura. Una mirada Crítica. Revista Latinoamericana de Estudios Educativos (Colombia), I(1), 125-147. https://www.redalyc.org/ pdf/1341/134116845008.pdf

Colmenares, S. (2010). La simplificación como problema complejo: Habraken y el S.A.R. En Com-densidad, estrategias de actuación urbana en áreas de baja densidad (pp. 1-10). Mairea Libros.

Consejo Interuniversitario Nacional (CIN). (2004, 27 de agosto). Primeras Jornadas de Reflexión sobre la Educación Superior en la Argentina, Horco Molle. Tucumán, Argentina. www.cin.edu.ar/ download_b.php?file=ANEXOAP537. doc

Deleuze, G. (1987). Foucault. Paidós.

Galvis, J. (2020). Del higienismo a la acupuntura urbana. Metáforas médicas y urbanismo excluyente en Bogotá. Territorios, (42), 1-29. https://www. doi.org/10.12804/revistas.urosario. edu.co/territorios/a.7297

García Ramírez, W. (2012). Arquitectura participativa: las formas de lo esencial. Revista de Arquitectura, 14(1), 4-11. http://editorial.ucatolica.edu.co/ojsucatolica/revistas_ucatolica/index. php/RevArq/article/view/721/736 
González, G. R., \& González, M. (2003). Universidad, sociedad y extensión universitaria: apuntes para un análisis.

Gutiérrez, R., \& Gutiérrez, V. R. (2012). Una mirada crítica a la arquitectura latinoamericana del siglo Xx. De las realidades a los desafíos. En E. Klein (Ed.), 1810-1910-2010, Independencias dependientes. Art and national identities in Latin America (pp. 1-16). https:// www.ugr.es/ rgutierr/PDFl/17l.pdf

Hernández Gálvez, A. (2014, 4 de noviembre). Los trabajos y los días del arquitecto en diario digital. Arquine. https://www.arquine.com/los-trabajos-y-los-dias-del-arquitecto/

Jara, O. (2017). La concepción metodológica dialéctica, los métodos y las técnicas participativas en la educación popular. Centro de Estudios y $\mathrm{Pu}-$ blicaciones Alforja. http://beu.extension.unicen.edu.ar/xmlui/bitstream/handle/123456789/245/ c on cepci \% C $3 \%$ B 3 n \% 20 m e t o d o $1 \%$ C $3 \%$ B 3 g i c a $\% 20$ dial\%C3\%A9ctica.pdf? sequence $=1$

Lameda Luna, H. (2014). Historiografía de la Arquitectura Latinoamericana. Del positivismo a la autocritica (18602010). Trienal de Investigación, Facultad de Arquitectura y Urbanismo, Universidad Central de Venezuela. http://trienal.fau.ucv.ve/2014/cd/ PDF/hyp/HP-17.pdf

Lastra, E. (2015, 1 al 3 de septiembre). Taller 11-Colonia Lola. En Ier Encuentro
Internacional. Taller Total: La Formación Universitaria y la Dimensión Social del Profesional (pp. 381-391). Universidad Nacional de Córdoba. https://issuu.com/arquimedesfederico/docs/0000001_publicaci__n

Lefebvre, H. (2013). La producción del espacio. Capitán Swing.

Lerner, J. (2005). Acupuntura Urbana de Jaime Lerner. Institut d'Arquitectura Avançada de Catalunya.

Liernur, J. F. (2001). Arquitectura en la Argentina del siglo XX: La construcción de la modernidad. Fondo Nacional de las Artes.

Lydon, M. (2012). Urbanismo Táctico 2. Acción a corto plazo, cambio a largo plazo. The Street Plans Collaborative. https://issuu.com/streetplanscollaborative/docs/urbanismo_tactico_2_digital_edition

Maass, M., \& Sabulsky, G. (2015). La vinculación como estrategia de formación en la educación superior. Áreas de Humanidades y Ciencias Sociales y de Artes, Arquitectura y Diseño en La vinculación como estrategia de formación en educación superior Sistematización de experiencias y buenas prácticas de docentes universitarios. Red Innova Cesal, 85-144. http:// www.innovacesal.org/micrositio_redic_2015/redic_pub2015_integrado. pdf\#page $=86$

Márquez López, L., \& Pradilla Cobos, E. (2016). Los territorios latinoamericanos territarios 44 Especial

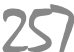




\section{territorias} 44 Especial en la mundialización del capital. Territorios, (34), 17-34. https://doi. org/10.12804/territ34.2016.01

Miranda Gassull, V. (2017). El hábitat popular. Algunos aportes teóricos de la realidad habitacional de sectores desposeídos. Territorios, (36), 217-238. https://doi.org/10.12804/revistas. urosario.edu.co/territorios/a.4440.

Miranda Gassull, V. (2017). Hábitat de producción social en tierras secas del norte de la provincia de Mendoza (Tesis doctoral). Universidad Nacional de Cuyo.

Montaner, J. M. (1999). Arquitectura y crítica. Editorial Gustavo Gilli.

Ortiz Flores, E. (2012). Producción Social de la Vivienda y el Hábitat. Bases conceptuales y correlación con los procesos habitacionales. Programa Regional de Vivienda y Hábitat (13-44); Centro Cooperativo Sueco de San José; Ediciones Trilce.

Revista Los Libros. (1971, noviembre). La experiencia del Taller Total. Revista Los Libros, para una crítica politica de la cultura, 23(7).

Robinson, J. (2006). Ordinary cities: Between Modernity and Development. Routledge.

Romero, G. (2002). La producción social del hábitat. Reflexiones sobre su historia, concepciones y propuestas. En E. Ortiz Flores \& L. Zarate (Comps.), Vivitos y coleando. 40 años trabajando por el hábitat popular de América Latina. UAM.
Romero, G., \& Mesías, R. (2004). La participación en el diseño urbano arquitectónico en la producción social del hábitat. Publicación del Programa Iberoamericano de Ciencia y Tecnología para el Desarrollo CYTED.

Romero, G., Salceda, J., Hernández, J., \& Castañeda, U. (2018). El diseño participativo. De la crítica a la praxis. En M. Hernández \& V. García (Eds.), Visiones del hábitat en América Latina: participación, autogestión, habitabilidad (Vol. 32) (pp. 123-139). Editorial Reverté.

Salinas, F. (1970). La arquitectura revolucionaria del Tercer Mundo. En R. Segre \& F. Salinas, Ensayos sobre arquitectura e ideología en Cuba revolucionaria (p. 15). Universidad de la Habana.

Segre, R. (1987). América Latina en su arquitectura (7a. ed.). Siglo XXI Editores. Tauber, F. (2010). La universidad argentina en el Bicentenario. Discurso de asunción a la Presidencia de la UNLP, periodo 2010-2014. La Plata, Buenos Aires, Argentina. http:// sedici.unlp.edu.ar/bitstream/handle/10915/44416/Versi\%C3\%B3n_2. pdf? sequence $=3$ \& isAllowed $=y$

Tommasino, H., \& Cano, A. (2016). Modelos de extensión universitaria en las universidades latinoamericanas en el siglo XXI: tendencias y controversias. Revista Universidades, 67, 7-24. https://cutt.ly/DxN7vxa

Universidad de la República (UDELAR). (2009). Para la renovación de la 
enseñanza y la curricularización de la extensión y las actividades en el medio. Resolución del Consejo Directivo Central de la Universidad de la República, UDELAR.

Utgar Salceda, J. (2020). Curso de Diseño Participativo. HIC-LA-UNAM.

Verdaguer Viana-Cárdenas, C. (2002). Por un urbanismo de los ciudadanos.
Boletin $C F+S, 24$. http://habitat. aq.upm.es/boletin/n24/acver.html

Waisman, M. (1972). La estructura histórica del entorno. Ediciones Nueva Visión SAIC.

Waisman, M. (1989). Para una caracterización de la arquitectura latinoamericana. Arquitecturas del Sur, 5(14), 8-10. http://revistas.ubiobio.cl/index.php/ AS/article/view/992 